\title{
Presupuestos metafísicos de una sana historiografía crítica aplicada al texto bíblico
}

\author{
CARLOS CASANOVA \\ Centro de Estudios Tomistas, Universidad Santo Tomás (Chile) \\ ccasanovag@santotomas.cl
}

\begin{abstract}
Resumen
Trata sobre los presupuestos metafísicos de aceptar la Biblia como Palabra de Dios. En particular, trata sobre la posibilidad de las intervenciones divinas, de los milagros y profecías. Responde al argumento de Hobbes por el determinismo, al principio de la clausura causal del mundo, a la crítica de Hume a la posibilidad de probar un milagro y a la negación de las profecías.

Palabra claves: Biblia, historicidad del Nuevo Testamento, intervenciones divinas, milagros, profecías.
\end{abstract}

\section{Metaphysical presuppositions for a sound critical historiography applied to the biblical text}

\begin{abstract}
This paper deals with the metaphysical presuppositions which underlie the acceptance of the Bible as the Word of God. In particular, it deals with the possibility of divine interventions, miracles and prophecies. It answers to the Hobbesian argument for determinism, to the principle of the causal closure of the world, to Hume's criticism of the possibility to prove miracles and to the negation of prophecies.
\end{abstract}

Key words: Bible, historicity of the New Testament, divine interventions, miracles, prophecies.

Doctor en Filosofía por la Universidad de Navarra. Profesor Titular de la Universidad Santo Tomás. Coordinador de Investigación y Publicaciones del Centro de Estudios Tomistas. Ha publicado: Dios, el ser y la ciencia, según Aristóteles (2007), El hombre. Frontera entre lo inteligible y lo sensible (2010), Fisica y realidade (2013), Racionalidad y justicia (2013), y numerosos artículos. Entre ellos: "Sobre la inadecuación de la filosofía hermenéutica para explicar la revelación divina” (2015).

Recibido: 29/Octubre/2015 - Aceptado: 11/Diciembre/2015 
La perspectiva que normalmente asume la escuela histórico-crítica de exégesis bíblica presenta una serie de problemas que deberían ser considerados metafísicamente ${ }^{1}$. El primero de esos problemas es que supone que la historia es una ciencia, y que está sujeta al método científico. Ambas afirmaciones son inexactas. La historia no es ciencia en el sentido de conocimiento de causas o principios universales cuya operación pueda ser comprobada por experimentos o demostraciones. Es una disciplina, eso sí, que intenta establecer la verdad. Pero, puesto que se refiere a acontecimientos singulares y pasados, debe reposar en la fe en ciertos testimonios. Además, como no puede examinar todas las fuentes y debe partir de una cierta perspectiva, su enfoque principal no procede de un análisis dialéctico y cuidadoso de los acontecimientos particulares sobre los que investiga (aunque pueda ser afectado por ellos), a diferencia de lo que ocurre, según Isaac Newton, con los principios de la mecánica. El enfoque principal de la historia procede de una reflexión sobre la realidad que tiene otra naturaleza: es, o bien una investigación filosófica o análoga (propia o ajena), o una disposición teológica (guiada por la luz de la Fe), o una ideología. Por otra parte, la historia no puede seguir el método científico, precisamente porque sus hipótesis no proceden sólo de un análisis dialéctico de los acontecimientos investigados, ni pueden ser demostradas matemáticamente, ni verificadas o falsadas por experimentos en el sentido preciso de esta palabra.

Si se comprende bien lo anterior, salta a la vista un segundo problema. A menudo, en efecto, se hace un tratamiento errado de las fuentes no cristianas o no hebreas que se refieren al cristianismo primitivo o al contexto de la historia de Israel. Algunos suponen, por ejemplo, que contienen tal referencia debido a interpolaciones introducidas por copistas cristianos o por otros copistas con motivaciones similares. Se puede observar en general, en relación con este punto, que los diversos autores de las varias escuelas historiográficas críticas a menudo desestiman los escritos que deberían ser las fuentes de sus narraciones, y los verificadores de sus hipótesis, en virtud de que no cuadran (esos escritos) con dichas hipótesis. A menudo, pues, el carro va delante del caballo.

Es por esta razón, precisamente, que algunos desestiman los escritos del Nuevo Testamento como fuentes para conocer al Jesús histórico o que, en general, no atinan a leer adecuadamente los libros revelados. Estos críticos asumen una perspectiva naturalista, se basan en hipótesis

1 Este artículo recoge ideas que expuse en una ponencia presentada en las VII Jornadas Internacionales de Filosofía y Teoría de la Historia, organizadas por la Universidad Adolfo Ibáñez, y que tuvieron lugar entre los días 26 y 28 de agosto de 2015. 
filosóficas que la filosofía puede refutar y que ha refutado y, desde el siglo XIX, las aplican de manera sistemática al estudio de la Sagrada Escritura $^{2}$. A causa de esos prejuicios, por ejemplo, se ha abandonado la enseñanza tradicional de que los Evangelios sinópticos fueron escritos muy temprano, hacia el año 50, pues se supone que no es posible que Cristo predijera tan claramente la destrucción de Jerusalén y del Templo. Supuestamente habrían sido escritos después del año 70. Esto a pesar de que Flavio Josefo dice en su De Bello Judaico que al empezar la guerra muchos huyeron de Jerusalén a causa de una antigua profecía; a pesar de que hay profecías semejantes en Deuteronomio 4 y 28 y en Daniel; y a pesar también de que, como ha mostrado Vittorio Messori (s/f), se han encontrado fragmentos del texto hebreo del Evangelio según san Marcos en las cavernas del Qumrán. -Lo cual significa que había sido redactado dicho Evangelio antes del año 68; más aún: con técnicas papirológicas se ha datado hacia el año 50. Se atribuye una fecha tardía a la composición de los Evangelios por la razón señalada, a pesar, finalmente, de la multitud de profecías que se cumplieron en el propio Jesús ${ }^{3}$.

Lo que propongo no es rechazar la investigación historiográfica, sino ponerla sobre un fundamento más sólido y más teológico. Como ha dicho el amado Santo Padre Benedicto XVI (2011: 6-7):

Si la exégesis bíblica no quiere seguir agotándose en formular siempre hipótesis distintas, haciéndose teológicamente insignificante, ha de dar un paso metodológicamente nuevo volviendo a reconocerse como disciplina teológica, sin renunciar a su carácter histórico. Debe aprender que la hermenéutica positivista, de la que toma su punto de partida, no es expresión de la única razón válida, que se ha encontrado definitivamente a sí misma, sino que constituye una determinada especie de racionabilidad históricamente condicionada, capaz de correcciones e integraciones, y necesitada de ellas. Dicha exégesis ha de reconocer que una hermenéutica de la fe, desarrollada de manera correcta, es conforme al texto y puede unirse con una

2 Prejuicios semejantes, pienso yo, han llevado a aplicar criterios diversos en las ediciones críticas del Nuevo Testamento a los que se usan en la edición de otras obras griegas clásicas, como los poemas homéricos. En el caso de la Biblia se han preferido los manuscritos alejandrinos, más cortos, a los de la tradición griega principal, que es la bizantina. En el caso de Homero se ha preferido la tradición principal, que se halla, en su extensión, entre los extremos de la tradición popular (más larga) y la alejandrina (más corta). Por supuesto, se ha hecho esta diferencia con un pretexto científico, pero ese pretexto es, en este caso, una hipótesis que no parece suficientemente justificada: una supuesta dependencia de todos los manuscritos de la tradición principal, respecto de un hipotético acuerdo tomado en el siglo IV para unificar el texto (Maurice A. Robinson, 2001: en especial, nn. 2 y 11-23).

3 Piénsese en el Salmo 21 (Vulgata) o en el Poema del Siervo de Yavé.

VERITAS, No 34 (Marzo 2016) 
hermenéutica histórica consciente de sus propios límites para formar una totalidad nueva.

En este artículo quiero estudiar un par de los principales presupuestos metafísicos que permitirían una confluencia de la investigación histórica y la teológica. Usaré las interesantes observaciones contenidas en una serie de importantes documentos magisteriales que se dieron en el propio Concilio Vaticano II (Dei Verbum; n. 12, en particular), o durante el Concilio ("Sobre la verdad histórica de los Evangelios"); y tendré también en cuenta un documento promulgado después del Concilio (la Exhortación Apostólica Postsinodal Verbum Domini, de Benedicto XVI), para mostrar la conexión entre la exégesis y esos presupuestos. Todas estas declaraciones magisteriales subrayan tanto que el Nuevo Testamento es la principal fuente histórica para conocer a Jesús como la necesidad de acercarse al texto con Fe, para poder captar su sentido.

\section{El sentido literal y los géneros literarios}

La Constitución Dogmática Dei Verbum, en su n. 12, impone a los intérpretes el deber de encontrar, en primer término, el sentido literal de la Sagrada Escritura. Pero, recogiendo las enseñanzas de Divino afflante Spiritu, enseña que, para ello, es preciso investigar cuáles son los géneros literarios en los que se expresa la verdad divina en cada contexto: "histórico, profético, poético o en otros géneros literarios. [...] Pues para entender rectamente lo que el autor sagrado quiso afirmar en sus escritos, hay que atender cuidadosamente tanto a las formas nativas usadas de pensar, de hablar o de narrar vigentes en los tiempos del hagiógrafo, como a las que en aquella época solían usarse en el trato mutuo de los hombres".

Así, por ejemplo, una lectura atenta de los tres primeros capítulos del libro del Génesis puede mostrar que muchos aspectos no pueden interpretarse históricamente, sino cultualmente. La creación en siete días, por ejemplo, tiene, sin duda, un fin cultual. Asimismo, puede mostrar la conexión o la semejanza entre la narración de estos capítulos y narraciones míticas de diversos pueblos, especialmente de pueblos mesopotámicos o de Egipto. Pero, no debe dejar de resaltar la admirable diferencia: la unicidad de Dios, su trascendencia, el que Dios haya creado todo ("el cielo y la tierra"), etc. De esta manera, se puede distinguir el núcleo que requiere una interpretación histórica y el lenguaje mítico que lo reviste.

Por otra parte, el examen de los Evangelios y los Hechos revela que se trata de libros que tienen una indudable intención histórica, escritos en 
una época en que la disciplina histórica estaba bastante desarrollada. De aquí que el propio Concilio Vaticano II haya afirmado que:

La Santa Madre Iglesia firme y constantemente ha creído y cree que los cuatro referidos Evangelios, cuya historicidad afirma sin vacilar, comunican fielmente lo que Jesús Hijo de Dios, viviendo entre los hombres, hizo y enseñó realmente para la salvación de ellos, hasta el día que fue levantado al cielo. [...] Los autores sagrados escribieron los cuatro Evangelios escogiendo algunas cosas de las muchas que ya se trasmitían de palabra o por escrito, sintetizando otras, o explicándolas atendiendo a la condición de las Iglesias, reteniendo por fin la forma de proclamación de manera que siempre nos comunicaban la verdad sincera acerca de Jesús. Escribieron, pues, sacándolo ya de su memoria o recuerdos, ya del testimonio de quienes 'desde el principio fueron testigos oculares y ministros de la palabra' para que conozcamos 'la verdad' de las palabras que nos enseñan [cf. Lc., 1,2-4] (Concilio Vaticano II, 1965: \19).

Todavía más clara fue la Pontifica Comisión Bíblica (1964: n. 2), que en un documento contemporáneo al Concilio Vaticano II (21.04.64) se pronunció Acerca de la veracidad bistórica de los evangelios, y nos entregó observaciones de gran valor:

Fue esta instrucción primitiva, hecha primero de manera oral, y luego por escrito -porque pronto ocurrió que muchos intentaron "ordenar la narración de las cosas" que se relacionaban con el Señor Jesús-, lo que los autores sagrados, con el método conveniente al fin que cada uno se propuso, consignaron por medio de los cuatro evangelios para utilidad de las iglesias. Escogiendo algunas cosas entre las muchas entregadas, reduciendo otras en una síntesis, explicando otras con la atención puesta en el estado de las iglesias, se valieron de todo auxilio para que los lectores conocieran la firmeza de esas palabras en las que habían sido instruidos. Pues los hagiógrafos, de esas cosas que habían recibido, seleccionaron las que eran más adecuadas a las varias condiciones de los fieles y al fin que ellos [los mismos autores] buscaban. Puesto que el sentido de un enunciado depende también de la secuencia de los asuntos, los Evangelistas que entregaron las palabras y las acciones realizadas por el Salvador, las explicaron para utilidad de los lectores, uno en un contexto, otro en otro contexto. Por lo cual debe el exégeta indagar a qué apuntaba el Evangelista cuando narraba un dicho o un hecho de tal modo o poniéndolo en un contexto determinado. Pues que los Evangelistas refieran los dichos o las acciones realizadas por el Señor en un orden diverso, en lo más mínimo impide la verdad de la narración. Lo mismo puede decirse del expresar de modo diverso sus sentencias no al pie de la letra, pero reteniendo su sentido. Porque, como dice san Agustín: "Es muy probable que cada uno de los Evangelistas creyera que debía narrar en el orden en que quiso Dios sugerir a su recuerdo esas mis- 
mas cosas que narraba. -Por lo menos en esos asuntos en los que adoptar un orden u otro en nada disminuía la autoridad y la verdad evangélicas. Porque el Espíritu Santo, que distribuye sus bienes a cada uno como quiere, sin duda rigió y gobernó las mentes de los santos en lo que se refiere al recordar lo que debían escribir, para el bien de los Libros que habían de situarse en tan alta cumbre de autoridad, y permitió que ordenaran su narración, uno de esta manera, otro de esta otra, y que todo el que buscara con pía diligencia pudiera encontrar el divino auxilio" (De consensu Evang., 2, 21, 61s.; PL 34,1102).

El exégeta, a no ser que atienda a todas estas cosas que tienen que ver con el origen y la composición de los Evangelios, y emplee debidamente cuanto pueda probarse de lo que han producido las recientes investigaciones [quaetumque probanda recentes investigationes attulerunt], no cumplirá su oficio de escrutar cuál haya sido la intención de los hagiógrafos o qué hayan dicho realmente. Puesto que a partir de cuanto las nuevas investigaciones han arrojado se manifiesta que la doctrina y la vida de Jesús no han sido simplemente narradas con el único fin de que se conservaran en la memoria, sino que han sido "predicadas" de tal modo que dieran a la Iglesia fundamento de fe y de costumbres, el intérprete, al escrutar infatigablemente el testimonio de los Evangelistas, podrá ilustrar más profundamente la perenne fuerza teológica de los Evangelios [...].

\section{El sentido espiritual, encima de los géneros literarios. Dios co- mo Autor principal}

Una vez que se ha determinado el sentido literal de los textos, que puede ser un sentido metafórico, por ejemplo, o hasta cierto punto mítico, o cultual, o histórico, es preciso escudriñar cómo Dios puede haber querido usar como instrumentos para expresar otras cosas, aun a las personas, las palabras y las acciones a que se refieren los textos o en que consisten los textos.

Estas observaciones presuponen que ha de considerarse a Dios como Autor principal de la Escritura y de los acontecimientos narrados en ella o que cristalizan en ella. Esta actitud subyace a toda la concepción cristiana de la Revelación, de la Escritura y de la relación entre la Providencia divina y la historia, y afecta en su raíz la recepción del texto sagrado. Así lo expresa sucintamente el Concilio Vaticano II en la Constitución Apostólica Dei Verbum:

Y como la Sagrada Escritura hay que leerla e interpretarla con el mismo Espíritu con que se escribió para sacar el sentido exacto de los textos sagrados, hay que atender no menos diligentemente al contenido y a la unidad de toda la Sagrada Escritura, teniendo en cuenta la Tradición viva de 
toda la Iglesia y la analogía de la fe $\mathrm{fe}^{4}$. Es deber de los exegetas trabajar según estas reglas para entender y exponer totalmente el sentido de la Sagrada Escritura, para que, como en un estudio previo, vaya madurando el juicio de la Iglesia (Concilio Vaticano II, 1965: \12).

\section{Los obstáculos que se interponen en el camino de los exégetas}

Algunos exégetas encuentran dos obstáculos principales para aceptar estas declaraciones del Magisterio. El primero, es su falta de Fe. Sin la luz interior que es don divino y que protegemos con nuestra constante vigilia y oración (velady orad, para que no caigáis en tentación), no se pueden aceptar las narraciones de los milagros ni, muy especialmente, el relato de la Resurrección. -Eso a pesar de que las señales de credibilidad historiográfica que nos dan las fuentes son abundantes. Uno de los mejores estudios en este sentido es el del Obispo anglicano N. T. Wright, publicado en su libro The Resurrection of the Son of God, que quedó resumido en el segundo apéndice del libro de Antony Flew, There is a God (Flew, 2007: 195-213). La evidencia histórica unida a la argumentación es aplastante. Como dice Newman: "[...] los milagros cristianos se apoyan en una evidencia más fuerte que la que puede darse para cualquiera de los hechos históricos que creemos firmemente. Esto lo han sentido los infieles que, por ello, han sido conducidos a negar la admisibilidad hasta del testimonio más sólido, cuando se da en apoyo de acontecimientos milagrosos, para, de ese modo, librarse del único medio por el que puede probarse que han ocurrido" (Newman, 1907: n. 13). Sobre esos infieles hablaremos más abajo. Con todo, el acontecimiento de la Resurrección es tan increíble, que requiere del salto de la Fe.

Y allí está el segundo obstáculo. Porque nuestra Fe no es un fideísmo, si la Resurrección fuera un imposible, no sería una parte de aquélla. Las falsas doctrinas filosóficas del naturalismo que niega toda intervención de Dios en la historia han dañado la Fe de muchos, ya sea para llevarlos a pensar que la Fe no requiere de la verdad histórica ${ }^{5}$, o ya sea para llevarlos a caer en el agnosticismo o el deísmo. Pero, si Dios existe, y si

4 Para una breve explicación de esta noción, véase Siervas de los Sagrados Corazones, 2012.

5 Immanuel Kant es quien inspira muchas de estas posiciones. Él, como Averroes, sostiene que la filosofía se encuentra por encima de la religión (Kant, 1960: 12, prefacio a la segunda edición). Pero, a causa de la bancarrota metafísica en que Kant juzgó que Hume había dejado a la filosofía, (a diferencia de Averroes) el prusiano reduce lo esencial de la religión a un asunto de moralidad filosófica, a una extensión de la moralidad filosófica, para ser más precisos (1960: 3-6, Prefacio a la primera edición). Así, aunque un aspecto de la religión incluya una cierta fe en acontecimientos históricos, este aspecto debe subordinarse a la moralidad filosófica, según Kant.

VERITAS, No 34 (Marzo 2016) 
es Omnipotente, no se ve por qué no podría tomar una naturaleza humana, ni por qué no podría volver a unir el alma al cuerpo de esa naturaleza humana.

La Pontificia Comisión Bíblica (1964: n. 1) identificó muy precisamente los mencionados problemas:

Donde el caso lo permita, es lícito al intérprete católico investigar cuáles elementos saludables se hallen en "el método de la historia de las formas", que puedan usarse debidamente para alcanzar una más plena inteligencia de los Evangelios. Condúzcase sin embargo con circunspección porque se hallan a menudo, mezclados con este método, ciertos principios filosóficos y teológicos que no pueden probarse [hand probanda] y que no rara vez corrompen tanto el método como las conclusiones sobre el asunto literario. Ocurre que algunos de los fautores de este método, arrastrados por las opiniones prejuiciadas del racionalismo, se niegan a reconocer la existencia del orden sobrenatural y la existencia o aun la posibilidad de la intervención del Dios personal en el mundo, concretada en el auxilio [dado a los hombres] de una revelación propiamente dicha, de milagros y de profecías. Otros, a partir de una falsa noción de la fe proceden como si a esas cosas [lo sobrenatural] les fuera indiferente la verdad histórica, más aun como si la verdad histórica no pudiera conciliarse con aquéllas. Otros niegan como a priori la fuerza e índole histórica de los documentos de la revelación. Otros, finalmente, poco atentos a la autoridad de los Apóstoles en la medida en que son testigos de Cristo, y a su influjo en la primitiva comunidad, ensalzan el poder creador de esta comunidad. Cosas todas que no sólo se oponen a la doctrina católica, sino que también carecen de fundamento científico y son ajenas a los rectos principios del método histórico.

Como se ve, la aceptación de las verdades históricas que contiene una comprensión adecuada de la Fe presupone que se acepte al menos como posible el que Dios conoce los futuros contingentes o libres, y el que Dios actúa sobre el mundo, de maneras ordinarias o extraordinarias (como en los milagros). La negación de la presciencia divina y de la acción de Dios sobre la mente de los hagiógrafos tiene, entonces, varios efectos deletéreos en lo que se refiere a la interpretación de la Escritura: en primer lugar, ha llevado a negar la historicidad de los relatos bíblicos; en segundo lugar, a negar la unidad de la Sagrada Escritura, sobre todo, entre el Antiguo y el Nuevo Testamentos. Los "críticos" han caído en su mayor parte en la herejía de Teodoro de Mopsuestia a que se refiere santo Tomás repetidamente en el Comentario al Libro de los Salmos (2014, nn. 86785 y 86959). En tercer lugar, esa negación ha llevado al rechazo de la distinción patrística de sentidos, entre el sentido histórico o literal, el alegórico, el tropológico o moral y el anagógico, que, siguiendo a los 
Padres, estudia santo Tomás en la Suma teológica I q. 1, a. 10 (también, s/f $\mathrm{f}^{2}$ : aa. 9 , s. c. 4 ; y 10, ad $13 \mathrm{~m}$ ). En el fondo, se niega que Dios pueda con su Omnipotencia usar como signos de otras realidades no sólo los símbolos lingüísticos, sino las personas y las cosas. Sobre este punto, en el corpus del artículo citado, santo Tomás dictamina brevemente, pero fundado en sus demostraciones teológicas y filosóficas, que: "el autor de la Sagrada Escritura es Dios, en cuya potestad se encuentra que, para significar algo, ordene no sólo las palabras (cosa que también el hombre puede hacer), sino también las cosas mismas. Y, por ello, puesto que en todas las ciencias las palabras tienen significado, esta ciencia [sagrada] tiene algo que le es propio, es decir, que las mismas cosas significadas por las palabras también significan algo. Entonces, aquella primera significación por la que las palabras significan las cosas pertenece al primer sentido, que es el histórico o literal. Pero aquella significación por la que las cosas significadas por las palabras significan a su vez otras cosas, se llama sentido espiritual, que se funda sobre el literal, y lo presupone. Este sentido espiritual se divide en tres", es decir, en los otros sentidos ya señalados.

Es preciso examinar estos puntos: la posibilidad de que el Dios omnipotente intervenga en el mundo y nos dé una revelación, acompañada de milagros y profecías. El problema se desdobla en dos: la acción de Dios y la presciencia divina. Seguiremos esta estrategia: responderemos a los argumentos que se han dado contra las intervenciones divinas. Schleiermacher y Bultman no dan argumentos, simplemente suponen que "en nuestra época" no se puede creer en milagros o en lo sobrenatural. A ellos los dejaremos de lado, por eso. Nos fijaremos en los autores que dieron los argumentos a causa de los cuales pareció a Schleiermacher que "ya" no era posible creer en milagros: Hobbes, Hume y Kant, sobre todo ${ }^{6}$. No nos ocuparemos del positivismo ni de la hermenéutica filosófica, que son nuevas fuentes de la negación de las verdades bíblicas, porque éstos ameritan embarcarse en largas discusiones puramente metafísicas, de las que ya me he ocupado en otros lugares (Casanova, 2007, 2015). En cuanto a Kant, en este contexto su importancia reside en su supuesta refutación de todas las vías racionales para probar la existencia de Dios. Pero aquí no examinaremos este presupuesto de nuestra argumentación, que es la existencia de un Dios omnipotente. Sólo apuntaré que ya Rogelio Rovira (2010) ha completado una refutación en forma de este punto

6 Daniel von Wachter (2013: 547-557) nos ofrece un breve resumen de la aparición de la teología modernista y de sus raíces en Spinoza, Herman Samuel Reimarus e Immanuel Kant. Kant, a su vez, presupone a Hume, que presupone a Hobbes.

VERITAS, No 34 (Marzo 2016) 
de la obra kantiana, y ha mostrado que Kant presupone lo que quiere probar.

\subsection{Acción del Dios omnipotente}

Existen fundamentalmente dos grupos de razones para negar la intervención de Dios en la historia o en el mundo físico por medio de milagros. El primer grupo procede, por lo menos, del tiempo de Thomas Hobbes y niega la existencia de todas las causas libres, incluido Dios. El segundo grupo niega que puedan violarse las leyes naturales.

\subsubsection{En el mundo físico no intervienen seres libres}

Dentro del primer grupo voy a considerar dos tipos de argumentos. Uno metafísico, que fue popularizado por Hobbes, y otro físico.

\subsubsection{Empecemos por el argumento metafísico}

Hobbes postuló en Of Liberty and Necessity lo siguiente:

[ 31] En séptimo lugar, sostengo que ser una causa suficiente es ser una causa a la cual nada le falta de lo que es necesario para producir el efecto. La misma es también una causa necesaria. Porque si fuere posible que una causa suficiente no produjere el efecto, entonces faltaría allí algo de lo que era necesario para producirlo, y así la causa no sería suficiente. Pero, si es imposible que una causa suficiente no produzca el efecto, entonces una causa suficiente es una causa necesaria, porque se dice que produce un efecto necesariamente eso que no puede sino producirlo. Por esto, es patente que todo lo que se produce se produce necesariamente, porque todo lo que se produce ha tenido una causa suficiente para producirlo, pues de otro modo no habría sido [producido]; y, por tanto, también las acciones voluntarias son necesarias (Hobbes, 1840: 274-275).

Pero, si se analiza cuidadosamente este texto, uno encuentra una afirmación que es necesaria y otra que es enteramente arbitraria. Es un primer principio de la razón y, por tanto, una verdad necesaria, que todo cambio requiere de una causa proporcionada, suficiente para producirlo. Todo el edificio de la ciencia y la filosofía naturales se levanta sobre este fundamento. Si yo toco esta mesa y me da una descarga eléctrica, yo inmediatamente busco un conductor en la mesa, y una conexión a una fuente de poder eléctrico. No se me ocurre que la corriente pueda venir de la nada. Es posible que no haya una causa aparente y que eso me lleve, por tanto, a buscar una causa desconocida. Pero yo sé de antemano que 
tiene que haber una causa suficiente para que yo tenga la experiencia de la descarga eléctrica.

He ahí la afirmación necesaria contenida en el texto de Hobbes. Pero, en cambio, es una afirmación gratuita el que si se da una causa suficiente para producir un efecto deba seguirse necesariamente dicho efecto. La causa puede sufrir interferencia de otra causa, como han señalado Elizabeth Anscombe (1981) ${ }^{7}$ y Daniel von Wachter (2015); o puede tratarse de una causa contingente, como sostuvo Werner Heisenberg de las causas que subyacen a los fenómenos cuánticos ${ }^{8}$; o puede tratarse de una causa libre. Volviendo al ejemplo anterior: si encuentro el conductor, etc., sé que esa causa puede ser interferida: por ejemplo, poniéndome guantines aislantes. Puede haber, por tanto, una causa suficiente para producir un efecto y, sin embargo, es posible que no ocurra el efecto? De manera análoga, si encontrara una hoja de papel con una elegante demostración de un teorema geométrico, sabría que directa o indirectamente un ser humano ha escrito eso allí, y que debe saber geometría, aunque habría podido no escribir esa elegante demostración en esa hoja de papel.

Lo que digo ahora fue ya apuntado mucho antes por santo Tomás de Aquino, contra una antigua formulación del error que Hobbes repitió y popularizó:

A lo décimoquinto ha de decirse que no toda causa produce el efecto por necesidad, incluso si se trata de una causa suficiente. Porque la causa puede ser impedida, de manera que no se siga su efecto, como ocurre con las causas naturales, que no producen sus efectos por necesidad, sino como "en la mayor parte de los casos", porque en unos pocos casos sufren un impedimento. Así, entonces, aquella causa que hace que la voluntad quiera algo no conviene que haga esto por necesidad. Porque por la misma voluntad puede ponerse un impedimento, bien sea removiendo el tipo de consideración que lo induce a querer, o bien sea cosiderando lo opuesto, es decir,

7 Anscombe conoce que la mecánica cuántica es no-determinista, y sabe que, sin embargo, aun si la mecánica newtoniana no hubiera sido falsada en algunas áreas, el determinismo sería falso, porque las causas señaladas por la mecánica newtoniana pueden ser interferidas de manera que no haya regularidades humeanas en la naturaleza y porque hay agentes como los animales y los seres dotados de libre arbitrio, que introducen cursos de eventos no predecibles por la mecánica.

8 La contingencia de la causa no es reductible a la interferencia, sino que puede deberse a la materia, tanto del agente como del paciente (Metafísica Épsilon 3. Edición de Oxford de 1973).

9 Como dice Elizabeth Anscombe: “one certainly can ask: 'May there not be enough to have made something happen-and yet it not have happened?'” (Anscombre, 1981: 135).

VERITAS, No 34 (Marzo 2016) 
que esto que se propone como bueno en algún aspecto no es bueno $\left(\mathrm{s} / \mathrm{f}^{1}\right.$ : q. 6 ad 15m. Mi traducción).

\subsubsection{E1 argumento físico}

Este argumento toma varias formas. En general, se funda en un principio metafísico, que sería la clausura causal del mundo físico. Una de las formas que adopta es la que sostiene que la primera ley de la termodinámica excluye la intervención de la voluntad humana sobre el cuerpo y la intervención de Dios sobre el mundo. En efecto, dice el oponente, si la energía no se crea ni se destruye, sino que sólo se transforma, no sería posible que unos agentes espirituales introdujeran movimientos que no estuvieran ya contenidos virtualmente en la interacción de las causas físicas, porque en ese caso estarían introduciendo energía en el sistema.

A este tipo de objeción se han dado diversas respuestas. Una de ellas fue la de Robert Larmer. El punto que señala este filósofo canadiense es que el principio físico, derivado de la experiencia, sólo muestra que la energía total de un sistema físico cerrado no puede aumentar, sino que se conserva. (La formulación más general que he presentado en el párrafo anterior sería sencillamente falsa.) Pero, en realidad, la acción de un ser espiritual como la voluntad humana [y, podríamos añadir, como la Voluntad de Dios] es, precisamente, una intervención que procede de fuera del sistema. Esto no es excluido ni por la primera ni por la segunda ley de la termodinámica (Larmer, 1986; 2009: 553-555).

Yo me he ocupado también de este tipo de problema en relación con la voluntad humana, en respuesta a una objeción que opone John Searle a John Eccles (2004: 29-31). Searle aduce que la intervención de una mente incorpórea sobre los movimientos corporales implicaría una violación del principio físico según el cual la materia y la energía del universo físico son constantes, ni se crean ni se destruyen. Larmer introduce en este punto una importante precisión, como ya apuntamos. Pero, además, si se atiende a las experiencias de las que procede el principio, la termodinámica de Carnot y Hermann von Helmholtz y su evolución posterior, se comprenderá que la causalidad del libre arbitrio sobre el cuerpo es otro tipo de experiencia, y los principios que auténticamente proceden de la experiencia no pueden contradecirse: una ciencia particular reposa sobre la evidencia que ella puede reunir, pero no puede negar la evidencia en que se base otra ciencia particular o la filosofía. Cómo se relacionen los dos campos de experiencia en el futuro o cómo se han relacionado en el pasado es un tema sobre el que yo al menos puedo decir ahora sólo lo siguiente: a) es obvio que la termodinámica tiene al- 
gunos rasgos peculiares en los seres vivos (por ejemplo, Piaget, 1980: 328-329), y esto creo que se trata de un fenómeno análogo al observado por Erwin Schrödinger (1967: 3-35) ${ }^{10}$. Y b) que la acción del alma sobre el cuerpo no es la de un agente externo sobre un cuerpo extraño (en esto me hallo en desacuerdo con Eccles, pues no soy un dualista) y la acción de la voluntad no es física en absoluto (Casanova, 2010: 128, nota 277).

\subsubsection{Las leyes naturales excluyen los milagros}

El segundo grupo de razones para negar la intervención de Dios en el mundo ha sido estudiado cuidadosamente por Daniel von Wachter, y también ha sido respondido por otros muchos filósofos y teólogos ${ }^{11}$. El objeto principal de las críticas de este grupo es David Hume (1778: sección 10, "Of Miracles").

La argumentación original humeana es bastante interesante. No se trata de una argumentación metafísica, como algunos la han interpretado, sino de una argumentación, digamos “judicial". Nosotros sabemos de los milagros narrados en la Escritura gracias al testimonio de los hagiógrafos. Sin embargo, cualquier testimonio debe ser evaluado antes de ser recibido. En esa evaluación se considera la credibilidad del testigo y la verosimilitud de lo atestiguado. Pero, por fuerte que sea la credibilidad del testigo, "un milagro es una violación de las leyes de la naturaleza; y, puesto que una firme e inalterable experiencia ha establecido estas leyes, la prueba contra un milagro, por la misma naturaleza del hecho, es tan entera como sea posible imaginar cualquier argumento tomado de la experiencia". La inverosimilitud del milagro es tan grande, que no es razonable aceptar la autoridad del testigo, a menos que ésta sea tal que una mentira del testigo sea más inverosímil que el milagro. Como Hume considera imposible esto último, entonces, considera que ha refutado los relatos de milagros contenidos en la Escritura. Entre ellos menciona de

10 Allí se muestra que en el nivel celular uno debería esperar que se manifestaran perturbaciones cuánticas que harían imposible la regularidad presupuesta por la vida, pero que, sorprendentemente, no se manifiestan. A partir de aquí, él planteó todo un proyecto de investigación para la biología de la segunda parte del siglo XX.

11 Reinhard Hütter (2013: 1033) ha hecho una lista de autores que recientemente han refutado a David Hume: J. Houston en su Reported Miracles: A Critique of Hume (Cambridge University Press, Cambridge, 1994); David Johnson en su Hume, Holism and Miracles (Cornell University Press, Ithaca, 1999); John Earman en su Hume's Abject Failure: The Argument Against Miracles (Oxford University Press, Nueva York, 2000); Craig S. Keener en su Miracles: The Credibility of the New Testament Accounts (Baker Academic, Grand Rapids, 2011).

VERITAS, No 34 (Marzo 2016) 
manera especial "un muerto que vuelva a la vida" ["a dead man restored to life"].

Es curioso que un hombre como Hume formule este argumento. Porque él sostiene que las leyes naturales no son sino regularidades a las que estamos acostumbrados. Debería, entonces, decir, por lo menos, que un testigo creíble sería una razón para pensar que la experiencia recogida hasta un momento determinado no era completa y que quizá haya que revisar la formulación de la supuesta ley. De esta manera, Hume, al oponer esta crítica, incurre en una contradicción, cosa que uno encuentra con frecuencia en la obra de este celebrado filósofo, tal como ha señalado Antony Flew ${ }^{12}$.

Daniel von Wachter (2015) ha intentado destruir la fuerza del argumento aduciendo que los milagros no violan ni constituyen excepciones a las leyes naturales. Observa con razón que las leyes naturales no son regularidades de la naturaleza como piensa Hume, sino predicciones condicionales ("Todo evento del tipo $x$ será sucedido por un evento del tipo $y$, si nada más está actuando sobre $\left.x^{\prime \prime}\right)$, que se basan en tendencias reales del mundo físico ${ }^{13}$. Si uno entiende por milagro un acontecimiento que es inconsistente con la constitución natural [normal] del mundo (Newman, 1907: n. 7), podría aún estar de acuerdo con von Wachter en lo que se refiere a algunos milagros. Por ejemplo: que el mar se aparte del camino de los israelitas pudo ser causado por la aplicación de una fuerza espiritual, que no actúa normalmente: los espíritus pueden tocar los

12 Flew lo dice así: "There is, for instance, no trace of the thesis that causal connections and necessities are nothing but false projections of regularities onto nature in the notorious section 'Of Miracles' in the first Inquiry”' (Flew, 2007: 58). -Y dejemos ahora de lado el hecho de que la experiencia puede llevar a reformular las leyes naturales, como lo ha hecho innumerables veces.

13 Hace exactamente las mismas observaciones Anscombe (1981: 138-139). Von Wachter (2015: 59-60) habla de "tendencias del universo físico" porque él piensa que toda la materia corpórea forma una sola entidad, a la que se añaden los seres vivos, desde las plantas hasta Dios. Pero yo discrepo en este punto de él: la química nos muestra que hay multitudes de entidades inanimadas y que las tendencias que forman la base de las leyes naturales tienen como sujeto a esas entidades, no al universo físico como una totalidad.

Por otra parte, aquí debería introducirse una distinción entre las afirmaciones científicas que constituyen hipótesis matemáticas que salvan los fenómenos y otras afirmaciones científicas. Las primeras pueden no tener referencia directa o indirecta a poderes de las entidades naturales, como ocurría con la astronomía ptolemaica y probablemente ocurre con la física relativista. Las segundas pueden tener como "verificadores" los poderes de las cosas naturales, aunque de maneras diversas, dependiendo de muchos factores. Uno de los más importantes es si se trata de afirmaciones de ciencia natural matematizada o de ciencia natural causal (Casanova, 2007). 
cuerpos sin ser ellos tocados. De este modo, este milagro podría explicarse sencillamente por la intervención de un agente, sin necesidad de postular una excepción a las leyes naturales.

Sin embargo, ciertamente hay milagros que implican una suspensión de las leyes naturales (que no una violación). Ejemplos de éstos serían la resurrección de un muerto, la multiplicación de los panes, o la conversión del pan y del vino en el Cuerpo y la Sangre de Cristo. Sobre este tipo de milagro, hay que decir tres cosas. En primer lugar, que la propia inteligibilidad del mundo físico exige que su principio sea una Inteligencia trascendente. Esto se puede entender en los términos profundos en que lo entendió Platón en el Fedón o en el Timeo; o Aristóteles en Metafísica alfa élatton y Lambda 7; o Friedrich Nietzsche (1980: 48-49) ${ }^{14}$. Pero, se puede entender también en términos menos profundos, aunque igualmente poderosos, como los que presenta Antony Flew en su libro There is a God, con citas de los principales científicos del siglo XX (Flew, 2007: 95-112). Como quiera que se entienda, está claro que la sujeción de la naturaleza a leyes es parte de la inteligibilidad de aquélla. Así que las leyes mismas dependen de una inteligencia trascendente. Si, como hemos mostrado en la sección anterior, esa inteligencia puede actuar sobre el mundo, no se ve por qué no podría suspender una ley ${ }^{15}$. Y de aquí se sigue una respuesta directa a la objeción de Hume, que voy a expresar en las palabras de John Henry Newman:

Esta última objeción $[. .$.$] deriva su fuerza de la asunción de que un milagro$ es un fenómeno estrictamente incausado, una auto-originada violación de la naturaleza; y se resuelve refiriendo el acontecimiento a la acción divina, un principio que (no puede negarse) ha dado origen a obras que indican un poder al menos de la misma magnitud que requiere el milagro. Habiédonse encontrado así una causa adecuada, la objeción se desvanece, en lo que se refiere a la cuestión del poder, y faltaría considerar si el hecho anómalo es tal que admita ser referido al Ser Supremo. Porque si no pudiera referirse a Él con propiedad, seguiría siendo tan improbable como si se desconociera que existe un tal agente" (Newman, 1907: n. 15) ${ }^{16}$.

14 “La 'razón' en filosofía”, n. 5, in fine.

15 John Henry Newman dijo algo semejante: "A proof drawn from an interruption in the course of nature is in the same line of argument as one deduced from the existence of that course, and in point of cogency is inferior to it. Were a being who had experience only of a chaotic world suddenly introduced into this orderly system of things, he would have an infinitely more powerful argument for the existence of a designing Mind, than a mere interruption of that system can afford. A Miracle is no argument to one who is deliberately, and on principle, an atheist" (Newman, 1907: n. 11).

16 Pero, puesto que también puede probarse la existencia de al menos un espíritu puro perverso, como apunta Platón en el libro $\mathrm{X}$ de las Leyes, sería posible que

VERITAS, No 34 (Marzo 2016) 
Pero, Hume puede todavía oponer una réplica:

aunque el Ser a quien se adscribe el milagro sea Omnipotente, no por ello el hecho se hace ni un poquitín más probable, puesto que es imposible que nosotros conozcamos los atributos o las acciones de tal Ser, excepto a partir de la experiencia que tenemos de sus obras como se manifiestan en el curso usual de la naturaleza (Hume, 1778: n. 38).

Y a esta réplica puedo responder que vuelve circular el argumento de Hume $^{17}$. En efecto, según él: no se puede creer en una intervención de Dios sobre el mundo porque no podemos saber que exista un Agente divino. Ahora bien, no podemos saber que exista un Agente divino porque no conocemos la causalidad real y, como no conocemos la causalidad real, ponemos en su lugar la regularidad de los acontecimientos sensoriales. Pero, supuesta la definición de causa como antecedente regular, hay que excluir a priori que pueda haber una causa que sea distinta de las que aparecen en las regularidades a que estamos acostumbrados. Como Dios no se nos aparece sensorialmente, no puede aparecer en esas regularidades. Si se diera un acontecimiento inusual, habría que considerarlo como incausado, o esperar a que se repitiera de manera usual para conectarlo con su antecedente. En otro lugar he mostrado ya que la negación por parte de Hume de la experiencia de la causalidad y otras estructuras metafísicas no es una prueba de su empirismo, sino una implicación necesaria y analítica de su modo de entender la experiencia. Si, como $\mathrm{Hu}-$ me, postuláramos que nuestra experiencia es meramente sensorial, nos veríamos forzados a decir que no tenemos experiencia de todas esas realidades que son sensibles per accidens, como la causalidad (Anscombe, 1981: 137-138), es decir, la derivación de un ser a partir de otro (Anscombe, 1981: 136). Pero es evidente que nuestra experiencia no es puramente sensorial, sino también intelectual. Entonces, se rompe el círculo humeano si decimos que sí se conoce la causalidad, que esa causalidad a menudo no tiene que ver con regularidades, como muestra Elizabeth Anscombe (1981: 137-139), y que en los acontecimientos ordinarios también interviene, como causa última, el Agente divino, como muestran las vías tomistas para probar la existencia de Dios. Sobre Dios conocemos muchas verdades, no sólo a partir del "curso normal de la naturaleza”, sino también a partir de la estructura metafísica de las realidades

ocurrieran hechos contrarios al curso común de la naturaleza que pudieran ser causados por él.

17 Ya había apuntado esto Craig S. Keener en su Miracles: The Credibility of the New Testament's Accounts. 
sensibles y de la estructura moral de la vida de las personas ${ }^{18}$, por ejemplo.

Podemos concluir, entonces, que un milagro no es algo enteramente inverosímil o contrario a la razón, aunque tampoco sea algo que se pueda creer ligeramente. De hecho, y esta es la segunda cosa que hay que decir en relación con la objeción humeana, ningún fiel está obligado a creer otros milagros que los que se narran o se predicen en la Escritura. Estos milagros cuentan con un testimonio mucho mayor que cualquier otro. Un testimonio que es más fuerte que nuestro conocimiento de las leyes naturales. (Conocimiento, sea dicho de paso, que se ve forzado a corregirse a la luz de nuevas experiencias y el uso de instrumentos más precisos). Ese testimonio es el de Dios. Como sabemos, la Fe es, en la expresión de san Agustín repetida por Benedicto XVI/Francisco I, "una palabra que resplandece en el interior del hombre" (2013: n. 33). Es decir, una luz sobrenatural que nos da Dios y que reverbera en los signos sensibles en que se concreta la profecía, principalmente en las palabras del Texto Sagrado.

Puede decirse, en tercer lugar, que los testigos humanos de los milagros nos cuentan lo que ellos han visto y oído: es decir, que Jesús, un hombre de cuya muerte no puede dudarse porque su corazón fue traspasado con una lanza, salió al tercer día del sepulcro y caminó, conversó y comió con sus discípulos. Uno de esos testigos fue san Pablo: es decir, un hombre que de ninguna manera podía estar inclinado a engañarse a sí mismo sobre esto, y cuya solidez intelectual y moral es superior a la de la gran mayoría de los hombres. Si, como hemos dicho, los milagros no son imposibles, no resulta irracional creer estos testimonios. A lo cual podría añadirse lo que dice santo Tomás: "sería mayor milagro que todos los signos pasados el que, sin signos milagrosos, el mundo hubiera sido llevado a creer por hombres simples y sin rango cosas tan arduas y a realizar obras tan difíciles y a esperar cosas tan altas [como las contenidas en la doctrina cristiana]" (Suma contra gentiles I, 6. Uso el texto de "Traditio spiritualis Sacri Ordinis Predicatorum").

A estas tres cosas puede añadirse una cuarta: hoy también ocurren milagros. Los expedientes de las causas de beatificación y canonización están llenos de testimonios en este sentido. Pero el fiel es libre de creerlos o no. Todo aconseja mucha prudencia en esta materia, pero ningún argumento excluye la posibilidad de los milagros. Una experiencia intere-

18 Acerca de la estructura moral, Newman, 1907: sección 2. Newman considera aquí otras objeciones, de Voltaire y de Bentham: el milagro implicaría que Dios no ha hecho bien el universo físico. Newman responde que el universo tiene otras dimensiones, como la moral, que pueden requerir una intervención física de Dios.

VERITAS, No 34 (Marzo 2016) 
sante en este sentido es la de las apariciones de Lourdes, y un testimonio interesante es la novela The Song of Bernardette, escrita por Franz Werfel.

David Hume opone a los milagros en que se apoya el cristianismo por lo menos otras dos objeciones. Pero sobre ellas tendré que tratar en otra oportunidad, para no perder el hilo de mi exposición ${ }^{19}$.

\subsection{Presciencia de Dios}

Para alguien que conciba el tiempo como una "forma de la sensibilidad" en cuyo seno ocurre todo lo que existe, o como una sucesión de experiencias, o como una línea continua que rige a todo lo real y que es ella misma tan real como lo regido por ella, las profecías de predestinación o de presciencia aparecen como una mera imposibilidad. Pero debe precisarse que no es lo mismo la profecía que la predicción de un acontecimiento contenido de manera necesaria en causas presentes. Muchos modernos que creyeron en predicciones, no han creído en profecías. La profecía en cuanto reveladora de un acontecimiento futuro, debe decirnos lo que ocurrirá, sin verlo en sus causas naturales por medio de una deducción humana. La profecía más perfecta es la que nos dice un acontecimiento futuro que depende de causas contingentes o libres y que, por lo mismo, es imposible predecir a partir del conocimiento de las causas segundas. Para mostrar la posibilidad de las profecías es preciso, por tanto, mostrar que la naturaleza del tiempo es muy diferente de las imágenes que de ella sugieren la obra de Kant o de Hume o de Newton. Así como Leibniz tuvo razón al sostener que lo que llamamos "espacio" no es una entidad absoluta, una suerte de "caja vacía" en la que se encuentran los cuerpos, sino un "lugar" consistente en relaciones entre los cuerpos, de manera parecida la base real del tiempo y la naturaleza del tiempo mismo como medida, exigen que mostremos que detrás de ellas hay un hermoso entramado de relaciones entre seres substanciales y accidentales, cambios y mentes. No puedo tratar ahora todos los proble-

19 En una breve nota de pie de página puedo adelantar que en una de ellas Hume postula que los milagros que aduce cada tradición religiosa son pruebas contra los que aducen las otras tradiciones. Ante semejante objeción habría que decir que no es así: otras culturas poseen o alcanzan algún conocimiento de una parte del universo moral que nos es revelado en el cristianismo. Por ello, los milagros cristianos no excluyen las intervenciones directas o indirectas de Dios y de otros espíritus en las diversas tradiciones, sino que nos muestran el sentido último de todas ellas. Tolkien (2001: 7173; 2000a: 100-101; 2000b: 109-110) lo expresó de un modo hermoso, en relación con los mitos: los mitos de las diversas tradiciones alcanzan su realización en la Revelación cristiana. Por otra parte, para determinar cuál de las tradiciones rivales pueda estar más cerca de la verdad serían útiles investigaciones como las que cristalizan en el capítulo 6 del libro I de la Suma contra los gentiles de santo Tomás. 
mas y sub-problemas que rodean este tema, pero sí puedo, con base sobre todo en los libros IV (capítulos 10-14) y VI de la Física y en los comentarios de santo Tomás, señalar las líneas generales desde las que puede elaborarse una argumentación sólida y completa para responder a quienes niegan la presciencia divina. Expondré mi propia comprensión del tema, sin pretender que expongo la doctrina de otros autores, pero reconoceré mi deuda con ellos.

Como es bien sabido, Aristóteles captó que existe una conexión intrínseca entre el movimiento y el tiempo (Física IV 11. Edición Academia de Berlín, 1960). Sus observaciones metafísicas se mueven en un plano diferente al fenoménico que consideró Einstein para formular su teoría de la relatividad. Por lo mismo, las observaciones aristotélicas conservan su fuerza también hoy. Hay cuatro tipos de cambio o movimiento, según Aristóteles, tres accidentales y uno substancial:

Local, que es el primero y más propio.

Cuantitativo, de crecimiento y decrecimiento.

Cualitativo, de alteración.

Substancial, de generación y corrupción, que no es propiamente movimiento, pues es instantáneo.

Los otros tres tipos de cambio o movimiento presuponen al movimiento local. Este movimiento es el único que es propiamente continuo, y es el que da su base ontológica al tiempo (Física IV 14, 223a28-32; y 223b13-24), en primer lugar. El aumento y decrecimiento siempre son discretos. La alteración, también. La corrupción y la generación son instantáneos. Todos los cambios se dan cuando se acerca el agente, en el mundo sensible. Para que algo se caliente, la fuente de calor debe acercarse a lo calentado. Para que se genere un perro, el espermatozoide debe acercarse y penetrar el óvulo, etc. Entonces, el movimiento local está presupuesto en los otros movimientos del mundo sensible.

¿Qué es el tiempo? Es una medida del movimiento según un antes y un después (Física IV 11, 220a25-26). Lo medimos por medio de un movimiento (Física IV 12) que conocemos bien y que presuponemos como regular. Por ejemplo: los días son unidades de tiempo que se determinan con la rotación de la tierra: el completarse de una rotación, que es un movimiento, se toma como unidad para medir otros movimientos o duraciones. Los años son unidades que dependen de la traslación de la tierra (Física IV 14, 223b18-24). Los meses son o bien divisiones de la traslación o dependen de las fases lunares. Las horas y los minutos y segundos son divisiones del día, que se realizan con base en un movimiento constante y conocido, como el de la arena que fluye en un reloj de arena 
o el de las agujas de un reloj de péndulo, etc. Hay que entender el tiempo, entonces, en relación con el movimiento local. Como éste, el tiempo también es continuo. Esto se puede comprender si se piensa en el movimiento continuo de la aguja de un reloj mecánico. Ella nunca se detiene en ningún punto; nosotros, con nuestro intelecto, hacemos divisiones en su movimiento y así marcamos las horas y los minutos o segundos.

La medida del movimiento la pone el intelecto. Sin intelecto no habría medida alguna, ni de la distancia, ni del movimiento (Física IV 14, 223a16-28). Pero la medida de la distancia, una vez definida (como una barra de plomo guardada en París y llamada "metro"), permite medir algo que existe en acto, la longitud. En cambio, la medida del movimiento, el tiempo, mide algo que ya no existe en acto, la duración pasada de las cosas. La duración es real, el no ser simultáneamente. Pero el pasado no existe en sí (Física IV 10, 218a2-4). ¿Dónde existe el pasado? Solamente en el alma y en Dios. Si existiera sólo en el alma, estaría a disposición de un poder totalitario el cambiar el pasado, es decir, sería cuasiverdadero el argumento de O’Brien en la prisión del Ministerio del Amor de 1984 , y tendría razón Herbert McCabe en este mismo punto ${ }^{20}$. No tienen razón, O’Brien y McCabe, porque hay Dios y Él se encuentra en la Eternidad, como veremos.

Si el tiempo es medida del movimiento, y si hay miles de movimientos, ¿cómo podría haber un solo tiempo y simultaneidad? Éste es un difícil problema filosófico. Aristóteles percibió la solidaridad de todos los seres del cosmos, que es real, pero la explicó de modo equivocado, como algo que depende del movimiento de la primera esfera de los cielos (Física IV 12, en conexión con 5. También, 14, 223b13-24). Hoy sabemos que no existen las esferas concéntricas de Aristóteles y que no poseen la causalidad que él les atribuyó. Así que hay que explicar la unidad del tiempo de otra manera. Pero es indudable que hay un flujo universal del cosmos creado, que se da a causa del orden que Dios ha puesto en el mundo. Por esta solidaridad del cosmos es posible que cuando yo hable ustedes me escuchen, que mientras estoy aquí parado mi esposa se encuentre en casa cuidando a los niños, o la luna siga girando en torno al sol con la interferencia que introduce la Tierra en su curso, etcétera.

20 Benjamin Murphy una vez me escribió: "Herbert McCabe in Law, Love and Language explicitly states that Orwell was wrong to suppose that the past cannot be changed. For Orwell, of course, O'Brien is the villain, but McCabe says that O'Brien's position makes sense. Orwell's error, he thinks, was that because of his empiricism, he assumed that there was a strict boundary line between fact and interpretation. For Orwell then (as reconstructed by McCabe), the facts of history are settled, but someone may come up with a new interpretation of those facts. For McCabe, the facts themselves may change, and so after a revolution, it is legitimate to re-write history". 
Ese flujo universal es como un cono giratorio, que tiene un eje y fluye menos a medida que se acerca al punto superior del que mana ese eje. La eternidad no es una duración infinita, sino un no transcurrir. Se parece a la mente de un compositor, digamos Mozart, que, tal como cuentan Hadamard y Poincaré, se despertaba algunas noches hirviendo en inspiración y corría a su escritorio como para tomar el dictado de lo que estaba en su mente; o para desplegar en multitud de símbolos la pieza musical que él veía en un acto simple (Penrose, 1989: capítulo 10). Después, esa obra, escrita en el papel, sería interpretada por una orquesta, y desplegada así físicamente. En el auditorio, el público que conoce de música, podría escucharla y recomponer su unidad en la memoria, una vez que hubiera sido interpretada completa. De manera semejante, la eternidad, como decía Boecio, es "la posesión toda, simultánea y perfecta de una vida interminable” (Boecio, 1934, Libro V, prosa 6) ${ }^{21}$. En relación con el despliegue del mundo, es una idea instantánea, que no fluye, pero que da origen a todo lo que se relaciona con el flujo, o fluye. Volviendo a mi otra metáfora, ella constituye el punto del que brota el eje y el eje mismo. Los ángeles están más cerca de ese punto y por eso su duración es diferente de la nuestra: en ellos no hay movimiento local ni dependen sus cambios del movimiento local. Sin embargo, también ellos tienen una cierta duración sucesiva. Son como la pieza escrita en el papel, que, una vez producida, ya no fluye, pero sirve de modelo a lo que fluye. Nosotros sí sufrimos movimientos locales y sí dependemos de ellos en cierta medida y manera. Por eso estamos en el tiempo, en el flujo universal del cosmos. Nos parecemos a quienes asisten al concierto y pueden comprender su unidad tras haberlo escuchado. No somos un puro flujo, como los sonidos de los instrumentos, que son imagen de las cosas puramente sensibles. Nuestra inteligencia nos permite recogernos más allá del puro fluir. Por eso tenemos historia, un intermedio entre la eternidad de Dios o el evo de los ángeles y el puro fluir de lo sensible. Los seres inanimados son un puro transcurso. Los animales con memoria se acercan a nosotros, etcétera.

El tiempo no es, por tanto, una suerte de línea que se va desplegando físicamente. Es la profundidad de la duración del aspecto del cosmos que está sujeto al movimiento local, en cuanto es considerado o medido por el hombre. Pero esa profundidad de la duración que nosotros vemos desenvolverse está en la Mente de Dios como una Idea Ejemplar, que es participada por los ángeles que sirven de modelo inmediato para el cosmos visible. Ella es conocida por nosotros, vaga y parcialmente, a partir

21 Ya Aristóteles había señalado que lo que existe siempre no está en el tiempo (Física IV 12, 221b3-6).

VERITAS, No 34 (Marzo 2016) 
de la Revelación y a partir del flujo de las cosas, que se da siempre bajo el canon fijo de nuestros conceptos (sin los que no podríamos comprender los sujetos del cambio), que son destellos mediatos de la Eternidad.

En la modernidad se han intentado otras explicaciones filosóficas del tiempo, pero han fracasado. Por ejemplo, la explicación empirista de Hume, con sus sensaciones atómicas, es incapaz de dar cuenta de la experiencia de la sucesión continua. Hume sostuvo, en efecto, que la experiencia de la sucesión temporal surgía de la sucesión de experiencias (1896: I.II.3 $)^{22}$. Pero William James le objetó, con toda razón, que una sucesión de experiencias no equivale a la experiencia de la sucesión (1981: 590-592) ${ }^{23}$. Lo que ocurre en realidad es que nuestro intelecto capta el continuo que es el movimiento, o la duración, y que en su totalidad y en cada parte tiene una cierta "profundidad". -El presente no es un instante (Física IV 13), por extraño que pueda parecer. La medida de ese continuo, como decía, la pone el intelecto, que es donde existe el pasado en cuanto pasado. Kant, por su parte, subrayó que el origen de la medida del movimiento está en el intelecto, pero fue demasiado lejos en esta dirección: supuso que el tiempo era una suerte de coordenada que la mente imponía a la experiencia y que, por tanto, no tenía ningún fundamento real. En este punto el idealismo trascendental kantiano presenta tantas dificultades como en el resto de este sistema, y una más: la física ha desarrollado un concepto de tiempo diferente al newtoniano y lo ha hecho a partir de la experiencia. De este modo ha quedado claro que el tiempo tal como lo concebía Kant, de manera newtoniana, como un solo flujo sin diferencias de duración, no ordena toda la experiencia. Así, la única metafísica del tiempo compatible con la ciencia actual es la aristotélica que, sin embargo, como dije antes, no está atada a la teoría relativista, pues ésta no es sino una explicación matemática de los fenómenos.

Si se penetra en la realidad del tiempo, por tanto, se puede comprender que la presciencia no es algo imposible. Dios, como compositor de la sinfonía del cosmos, se encuentra fuera del fluir de su música y conoce todo lo que ella contiene. Pero la presciencia encierra, con todo, misterios insondables que no puedo abordar ahora, y que están conectados, muy especialmente, con la realización de acciones libres pecaminosas.

22 Debo la referencia a un coloquio que ofreció Sean Kelly ante el Departamento de Filosofía de la Universidad de Notre Dame.

23 Debo nuevamente la referencia a Sean Kelly. Kant, por supuesto, observó igualmente que el tiempo no es reductible a una sucesión de sensaciones, pero postuló que era una forma a priori de la sensibilidad. 


\section{Conclusión}

Mi conclusión es que no hay verdaderas razones que se opongan a aceptar la realidad de los milagros y las profecías. Sin embargo, el hombre animal no puede comprender las cosas que son del Espiritu de Dios (I Cor. 2, 14). Lo que ocurre en verdad es que nosotros, absorbida nuestra atención por aquello que ocurre habitualmente, nos resistimos a aceptar algo que rompa nuestra rutina. Queremos medirlo todo con nuestro limitado entendimiento y, peor aún, queremos convertir en regla del entendimiento las experiencias que nos son habituales. Haciendo eco al Libro de Job o a la sabiduría de Francisca Xaviera del Valle podemos preguntarnos: ¿es que acaso realmente sabemos por qué un cuerpo no atraviesa a otro cuerpo? ¿'Tenemos la menor idea de por qué las fuerzas físicas acompañan a los cuerpos o a los campos o a las ondas? La razón puede llegar a establecer que el mundo tiene que haber sido creado de la nada. La razón puede asimismo comprender que el alma humana es incorpórea y, por lo mismo, que sólo un poder creador puede completar la generación del hombre. ¿Por qué no podría ese poder creador lograr que un alma informara de nuevo su cuerpo (de ella)? ¿Por qué no podría ese poder creador unir a su Ser una naturaleza humana creada? ¿Sólo porque a nuestro ingenio le cuesta comprender aun que eso sea posible?

Santo Tomás observa que el hombre animal es no sólo el que vive hundido en la sensualidad, sino también el que no acepta otro juicio sino el que procede de la percepción sensorial, incluido el que sólo juzga con la razón filosófica (Aquino, s/f $\mathrm{f}^{3}$ : capítulo II, lección 3) ${ }^{24}$. Éste, en efecto, llevado de la arrogancia a la que puede dar lugar una falsa filosofía ${ }^{25}$ acostumbrada a someterlo todo al propio juicio, rechaza como sinsentido todo lo que no puede entender. Un buen ejemplo de hombre animal en este sentido es Immanuel Kant. Nótese, en efecto, el siguiente pasaje del Prefacio a la segunda edición de La religión dentro de los límites de la sola razón:

Cuando, por ejemplo, la virtud como habilidad en las acciones que se conforman al deber (de acuerdo con la legalidad de aquéllas) se llama virtus phaenomenon, y la misma virtud como una disposición duradera hacia tales acciones hechas por el deber (a causa de su moralidad) se llama virtus noumenon, estas expresiones se usan solamente a causa de las escuelas; mientras el asunto mismo se contiene, aunque con otras palabras, en los más popu-

24 Tuve noticia de este pasaje gracias a la referencia de Piotr Roszak (2014: 312).

25 Aristóteles y Platón, por ejemplo, sabían que el saber filosófico no puede responder a los interrogantes más cruciales de la vida. Por eso acaban apuntando al mito. Sobre Aristóteles, cfr. Fr. 668, en Rose, citado por Jaeger (1984: 368).

VERITAS, No 34 (Marzo 2016) 
lares sermones e instrucción de los niños, y es fácilmente comprendida. ¡Ojalá pudiera decirse tanto como eso acerca de los misterios concernientes a la naturaleza divina que se cuentan entre las enseñanzas religiosas, misterios introducidos en el catecismo como si ellos fueran enteramente populares, pero que, en último término, deben transformarse primero en conceptos morales, si es que han de llegar a ser comprensibles para todos! (Kant, 1960: $12-13)^{26}$

Un hombre sin duda de ingenio poco común, pero cuya metafísica es insostenible hoy (entre otras razones, porque la experiencia ha obligado a cambiar la concepción de espacio y de tiempo que, según Kant, estaba presupuesta en toda experiencia), a causa, en último término, de que sin necesidad aceptó el principio humeano que reduce la pasividad de la experiencia humana a mera sensación, ese hombre quiere reducirlo todo a cuanto puede percibir por los sentidos y organizar con sus "formas de la sensibilidad” y demás categorías mentales. Santo Tomás de Aquino dio en el clavo cuando apuntó por qué convenía que Dios revelara verdades que sobrepasan la capacidad de la razón natural:

Otra utilidad viene de allí también, esto es, la represión de la presunción, que es madre del error. Pues hay algunos que presumen tanto de su propio ingenio que juzgan que toda la naturaleza de las cosas puede caber en su intelecto, es decir, que estiman que es verdadero todo lo que se les muestra a ellos, y falso lo que a ellos no se les muestra. Entonces, para que el ánimo humano, liberado de esta presunción, alcance la modesta investigación de la verdad, fue necesario al hombre que se propusieran de manera divina algunas verdades que excedieran enteramente a su entendimiento (Summa contra Gentiles I 5, n. 4).

Y la Escritura da su base última a esa observación del Aquinate: Nosotros hemos recibido el Espíritu de Dios para que conozcamos las cosas que nos han sido donadas por Dios: cosas de las que también hablamos, no con palabras aprendidas de sabiduría humana, sino con palabras aprendidas del Espíritu, juzgando las cosas espirituales con medios espirituales (1Cor 2, 12-13).

\section{REFERENCIAS}

-Anscombe, E. (1981). Causality and Determination. En The Collected Philosophical Papers of G. E. M. Anscombe. Metaphysics and the Philosophy of Mind (pp. 133-147). Oxford: Basil Backwell.

26 Averroes no conoció una verdadera revelación divina y, ciertamente, no recibió el don de la Fe. Por tanto, no podemos decir de él que fuera un "hombre animal". 
-Aquino, T. de (2014). Comentario al Libro de los Salmos, Tomo I, RIL Editores, Santiago. También disponible en línea: http://www.corpusthomisticum.org/cps00.html. Fecha de consulta: 30 de septiembre de 2015.

-Aquino, T. de $\left(\mathrm{s} / \mathrm{f}^{1}\right)$. De Malo. Disponible en: http://www.corpusthomisticum.org/qdm06.html. Fecha de consulta: 7 de octubre de 2015.

-Aquino, T. de (s/f2). De Veritate, q. 12. Disponible en: http://www.corpusthomisticum.org/qdv12.html. Fecha de consulta, 30 de septiembre de 2015.

-Aquino, T. de $\left(\mathrm{s} / \mathrm{f}^{3}\right)$. In $I$ ad Corinthios. Disponible en: http://www.corpusthomisticum.org/c1c.html. Fecha de consulta: 1 de octubre de 2015.

-Benedicto XVI. (2010). Verbum Domini. Disponible en: http://w2.vatican.va/content/benedictxvi/es/apost_exhortations/documents/hf_benxvi_exh_20100930_verbum-domini.html. Fecha de consulta: 5 de octubre de 2015.

-Benedicto XVI (2011). Jesús de Nazaret. Desde la entrada en Jerusalén hasta la Resurrección. Madrid: Planeta-Encuentro.

-Boecio, Anicio (1934). Philosophiae consolationis. En: Corpus Scriptorum Ecclesiasticorum Latinorum. (Volumen XLVII). Leipzig: Academiae Literarum Vindobonensis

-Casanova, C. A. (2007). Reflexiones metafísicas sobre la ciencia natural. Santiago: RIL Editores.

-Casanova, C. A. (2010). El hombre. Frontera entre lo inteligible y lo sensible. Santiago: Ediciones UC.

-Casanova, C. A. (2015). Sobre la inadecuación de la filosofía hermenéutica para explicar la revelación divina. Anales de Teología, 17.1, 65-83.

-Concilio Vaticano II (1965). Constitución Dei Verbum. Disponible en http://www.vatican.va/archive

/hist_councils/ii_vatican_council/documents/vat-ii_const_19651118_deiverbum_sp.html. Fecha de consulta: 5 de octubre de 2015.

-Eccles, J. (2004). Mind. A Brief Introduction. Oxford: Oxford University Press.

-Flew, A. (2007). There is a God. New York: Harper One.

-Francisco I (2013). Lumen Fidei. Disponible en: http://w2.vatican.va/content/francesco/

es/encyclicals/documents/papa-francesco_20130629_enciclica-lumen-

fidei.html. Fecha de consulta: 5 de octubre de 2015.

-Hobbes, Th. (1840), Of Liberty and Necessity. En W. Molesworth (Ed.), The English Works of Thomas Hobbes. (Tomo IV, págs. 229-278). London: John Bohn.

Disponible

en: https://archive.org/details/englishworkstho28hobbgoog. Fecha de consulta: 7 de octubre de 2015. 
-Hume, D. (1778). An Enquiry Concerning Human Understanding. En Philosophical Essays Concerning Human Understanding. London: A. Millar. Tomado de la edición de 1777, y publicado aquí: http://www.davidhume.org/texts/ehu.php. Fecha de consulta: 30 de septiembre de 2015.

-Hume, D. (1896). A Treatise of Human Nature. Oxford: Clarendon Press. Dispobible en: http://oll.libertyfund.org/titles/342. Fecha de consulta: 1 de octubre de 2015.

-Hütter, R. (2013). University Education, the Unity of Knowledge -and (Natural) Theology: John Henry Newman's Provocative Vision. Nova et Vetera, English Edition, 11 (4), 1017-1056.

-Jaeger, W. (1984). Aristóteles, bases para la historia de su desarrollo intelectual. México: Fondo de Cultura Económica.

-James, W. (1981). Principles. Cambridge, Mass.: Harvard University Press.

-Kant, I. (1960). Religion Within the Limits of Reason Alone. New York: Harpertorch Books.

-Larmer, R. (1986). Mind-Body Interaction and the Conservation of Energy. International Philosophical Quarterly, 26, 277-285.

-Larmer, R. (2009). Divine Agency and the Principle of the Conservation of Energy. Zygon, 44 (3), 553-555.

-Messori, V. (s/f). Veinte letras para un misterio. Atlántida No. 16. Disponible aquí: http://www.conocereisdeverdad.org/website/index.php?id=3032, consultada el 11 de marzo de 2015.

-Nestle, E; Nestle E.; Aland, K.; Aland, B.; Karavidopoulos, J.; Martini, C. M. \& Metzger, B. M.; Institutum Studiorum Textus Novi Testamenti Monasterii Westphaliae (1998). Novum Testamentum Graece. Sttutgart: Deutsche Bibelgesellschaft.

-Newman, J. H. (1907). The Miracles of Scripture Compared with those reported elsewhere, as regards Their Nature, Credibility, and Evidence. En Two Essays on Biblical and Ecclesiastical Miracles. London: Longmans, Green and Company. Disponible en: http://www.newmanreader.org/works/miracles/. Fecha de consulta: 30 de septiembre de 2015.

-Nietzsche, F. (1980). El crepúsculo de los ídolos o Cómo se filosofa con el martillo. Madrid: Alianza Editorial.

-Penrose, R. (1989). The Emperor's New Mind. New York: Oxford University Press.

-Piaget, J. (1980). Biología y conocimiento. Ensayo sobre las relaciones entre las regulaciones orgánicas y los procesos cognitivos. Madrid: Editorial Siglo XXI.

-Pontificia Comisión Bíblica (1964). Sobre la verdad histórica de los Evangelios. Disponible

en http://www.vatican.va/roman_curia/congregations/cfaith/pcb_documen ts/rc_con_cfaith_doc_19640421_verita-vangeli_lt.html. Consultado el: . Traducción castellana disponible aquí: https://sanatanasioysangregorio.wordpress.com/biblia/. Consultada el: 5 de octubre de 2015. 
-Robinson, M. A. (2001). New Testament Textual Criticism: The Case for Byzantine Priority. Disponible

aquí:

http://rosetta.reltech.org/TC/vol06/Robinson2001.html, consultado el 14 de marzo de 2015.

-Rovira, R. (2010). "No hay más que tres modos de probar, por razón especulativa, la existencia de Dios". Crítica de la clasificación kantiana de las pruebas teístas. En C. A. Casanova (ed.), El amor a la verdad, toda verdad y en todas las cosas. Ensayos en honor a Josef Seifert (pp. 157-180). Santiago: Ediciones $\mathrm{UC}$.

-Roszak, P. (2014). Exégesis y metafísica. En torno a la hermenéutica bíblica de Tomás de Aquino. Revista Salmanticensis, LXI (2), 301-323.

-Schrödinger, E. (1967). What Is Life? Cambridge at the University Press.

-Siervas de los Corazones Traspasados de Jesús y María. (2012). Voz: "Hermenéutica. Interpretación de la Biblia". En Pequeña enciclopedia católica. Disponible en: http://www.corazones.org/diccionario/ diccionario_2012/a_diccionario_catolico.html. Fecha de consulta: 5 de octubre de 2015.

-Tolkien, J. R. R. (2001) On Fairy Stories. En Tree and Leaf, Mythopoeia, The Homecoming of Beorbtnoth Beorbthelm's Son (pp. 1-81). London: Harper.

-Tolkien, J. R. R. (2000a). "Letter to Christopher Tolkien November $7^{\text {th- }} 8^{\text {th }}$ 1944". En Letters of J. R. R. Tolkien (H. Carpentr, Ed., pp. 99-103). New York: Hucghton Mifflin Company.

-Tolkien, J. R. R. (2000b). "Letter to Christopher Tolkien January 30th 1945”. En Letters of J. R. R. Tolkien (H. Carpentr, Ed., págs.108-111). Nueva York, Hucghton Mifflin Company).

-Wachter, D. von. (2013). Protestant Theology. En C. Meister and P. Copan (eds.), Routledge Companion to the Philosophy of Religion (pp. 547-557). New York: Routledge.

-Wachter, D. von. (2015). Los milagros no violan las leyes naturales. En C. A. Casanova (Ed.), El alma, la providencia y el Derecho natural (un ejercicio de filosofía como capacidad de juzgar) (págs 51-62). Santiago: IAP-Ril Editores.

Sumario: Introducción; 1. Sentido literal y géneros literarios; 2. El sentido literal por encima de los géneros literarios. Dios como Autor principal; 3. Los obstáculos que se interponen en el camino de los exégetas; 3.1. Acción del Dios omnipotente; 3.1.1. En el mundo físico no intervienen seres libres; 3.1.1.1. El argumento metafísico; 3.1.1.2. El argumento físico; 3.1.2. Las leyes naturales excluyen los milagros; 3.2. Presciencia de Dios; Conclusión; Referencias. 